\title{
El judaísmo y la cuestión de la guerra en la crisis final del segundo templo
}

\author{
César Vidal Manzanares */**
}

En buena medida puede decirse que el período que, convencionalmente, se conoce como Segundo Templo se halla enmarcado entre dos guerras. Su punto de arranque lo constituye el final del destierro babilónico vinculado al conflicto bélico contra Nabucodonosor II; su punto de conclusión se encuentra en la guerra contra Roma iniciada en el $66 \mathrm{~d}$. de $\mathrm{C}$. En el curso de esta última, Israel desaparecería como entidad política semi-independiente, el Templo de Jerusalén sería arrasado y la nación se vería sumida en un marasmo de consecuencias difícilmente exagerables. Lejos, por lo tanto, de tratarse de un fenómeno abordable desde una perspectiva meramente especulativa, la guerra constituyó una realidad palpable y una referencia constante en este marco cronológico, especialmente en su tramo final. No sólo eso. De las respuestas que se dieron a esta coyuntura dependió, de manera directa, no sólo el desarrollo ulterior de la política en Eretz Israel, sino, muy especialmente, el futuro del judaísmo y del cristianismo.

El judaísmo del Segundo Templo constituyó ideológicamente una realidad paradójica. Lo primero que llama la atención a la persona acostumbrada a las definiciones dogmáticas rígidas es su enorme flexibilidad doctrinal. Salvo la creencia en un Dios único que se había revelado históricamente al pueblo de Israel (Deuteronomio 6, 4) y cuyas palabras habian sido entregadas en la Torah a Moisés, era mucho más lo que separaba a las distintas sectas judías que lo que las unía. Pese a todo, seguía existiendo una consciencia clara de formar una unidad, la de Israel. En este estudio nos acercaremos a las diferentes escuelas religiosas (o sectas) judías centrándonos precisamente en su actitud peculiar frente a la guerra y en la manera en que esto influyó en su historia posterior.

* Departamento de Prehistoria e Historia Antigua. UNED.

** Ciclo Guerra y Religión en el Próximo Oriente y Egipto. 


\section{LOS FARISEOS ${ }^{2}$}

Los datos de que disponemos acerca de los fariseos nos han llegado fundamentalmente a partir de tres tipos de fuentes: los escritos de Josefo, los contenidos en el Nuevo Testamento y los de origen rabínico. Los primeros constituyen un retrato dirigido, fundamentalmente, a un público nojudío y, precisamente por ello, en su deseo por hacerse inteligible opacado en ocasiones en su exactitud. Por otro lado, Josefo mismo estaba ligado a los fariseos e incluso tenía un especial interés en que los romanos los aceptaran como la columna vertebral del pueblo judío tras la destrucción del Templo en el $70 \mathrm{~d}$. de C. No debería extrañarnos, por ello, que el retrato que nos transmite sea, lógicamente, muy favorable ${ }^{3}$ (Guerra 2, 8, 14; Ant. 13, 5, 9; 18, 1, 3), aunque no exento de ciertas contradicciones. Así, la descripción de las Antigüedades (escritas c. 94 d. de C.) contiene un matíz político y apologético que no aparece en la de la Guerra (c. $75 \mathrm{~d}$. de C.). En Ant 18, 1, 2-3, son presentados como todopoderosos (algo muy tentador, seguramente, para el invasor romano) aunque es más que dudoso que su popularidad entre la población fuera tan grande. El mismo relato de la influencia de los fariseos sobre la reina Alejandra (Ant, 13, 5, 5) o cerca del rey Herodes (Ant 17, 2, 4) parece estar concebido para mostrar lo beneficioso que podía resultar para un gobernante que deseara controlar Judea el tener a los fariseos como aliados políticos. En esta misma obra, Josefo retrotrae la influencia de los fariseos al reinado de Juan Hircano (134-104 a. de C.). La autobiografía de Josefo, titulada Vida, escrita en torno al $100 \mathrm{~d}$. de C., vuelve a abundar en esta presentación de los fariseos (Vida 38 y 39).

Aunque es innegable el tono laudatorio con que Josefo contempla a los fariseos, exagerando seguramente su popularidad y su influencia, lo cierto es que, pese a todo, nos proporciona algunas referencias sustanciales acerca de ellos mismos. Así nos permite saber que creían en la libertad humana, en la inmortalidad del alma, en un castigo y una recompensa eternos, en la resurrección, en la obligación de obedecer su

2 Sobre los fariseos, ver: L. FILKEnSTEIN, The Pharisees, Filadelfia, 1966; J. NEUSNER, The Rabbinic Traditions About the Pharisees Before 70, 3 vols, Leiden, 1971; J. BowkER, Jesus and the Pharisees, Cambridge, 1973; C. Vidal Manzanares, El Primer Evangelio: el Documento $Q$, Barcelona, 1992; Idem, Los esenios y los rollos del mar Muerto, Barcelona, 1992; Idem, "Fariseos" en Diccionario de las tres religiones monoteístas: judaísmo, cristianismo e islam, Madrid, 1992; Idem, "Fariseos» en Diccionario de Jesús y los Evangelios, Estella, 1994.

3 Las referencias completas a los fariseos pueden encontrarse en C. VIDAL MANZANARES, El Primer Evangelio: el Documento $Q$, Barcelona, 1992, c. 7. 
tradición interpretativa referida a obligaciones religiosas como las oraciones, los ritos de adoración, etc. Además estaban dispuestos (seguramente, no sólo eso) a obtener influencia política en la vida de Israel. Naturalmente, a estas notas distintivas habría que añadir la común creencia en el Dios único y en su Torah; la aceptación del sistema de sacrificios sagrados del Templo (que, no obstante, no era común a todas las sectas) y la creencia en la venida del Mesías (que tampoco era sustentada por todos).

El Nuevo Testamento ofrece un retrato de los fariseos que, a diferencia del presentado por Josefo, no arranca de una disposición favorable a los mismos. El Evangelio de Mateo, en especial, muestra una notable animadversión hacia ellos. Si efectivamente su autor fue el antiguo publicano llamado Leví o Mateo, o se utilizaron tradiciones recogidas por el mismo, podría explicarse tal oposición en el recuerdo del desprecio con que fue contemplado durante años por aquellos «que se consideraban a si mismos justos».

Aunque Jesús parece haber reconocido (MATEO 23, 2-3) que mucho de lo que decían era adecuado, se manifestó opuesto a las interpretaciones farisaicas en cuestiones como el cumplimiento del sábado (MATEO 12, 2; Marcos 2, 27), los lavatorios de manos antes de las comidas (LUCAS 11, $37 \mathrm{ss}$ ), sus normas alimenticias (MARcos 7, $1 \mathrm{ss}$ ) y, en general, todas aquellas tradiciones interpretativas que tendían a centrarse en el ritual desviando con ello la atención de lo que él consideraba lo esencial de la ley divina (Mateo 23, 23-4). Para él, resultaba intolerable que hubieran "sustituido los mandamientos de Dios por enseñanzas de hombres" (MATEO 15, 9; Marcos 7, 7).

Las tradiciones rabínicas sobre los fariseos se hallan recogidas en la Mishnah (concluida hacia el $200 \mathrm{~d}$. de C. aunque sus materiales son muy anteriores), la Tosefta (escrita hacia el $250 \mathrm{~d}$. de C.), y los dos Talmudim, el palestino (escrito sobre el 400-450 d. de C.) y el babilonio (escrito hacia el 500-600 d. de C.). Dada la distancia considerable de tiempo entre estos materiales y el periodo de tiempo abordado, los mismos han de ser examinados críticamente. J. Neusner ${ }^{4}$ ha señalado la existencia de 371 tradiciones distintas, contenidas en 655 pasajes, relacionadas con los fariseos anteriores al año $70 \mathrm{~d}$. de C. De las 371 , unas 280 están relacionadas con un fariseo llamado Hillel. El mismo fue un rabino del s. I a. de C. que vino desde Babilonia hasta Judea y fundó una escuela de interpretación

4 J. NEUSNER, From Politics to Piety: The Emergence of Rabbinic Judaism, Nueva York, 1979,
p. 81 . 
concreta. Opuesta a la escuela del rabino Shammai, se convertiría en la corriente dominante del fariseismo ( $y$, con ello, del judaísmo) a finales del s. I d. de C.

En no pequeña medida el retrato que los Evangelios ofrecen de los fariseos, se ve corroborado por testimonios de las fuentes rabínicas en buen número de casos y es coincidente en aspectos doctrinales con el que vemos en Josefo. De hecho, no deja de ser interesante el que el Talmud (SOTAH 22b; J. BERAJOT 14 b) al citar las siete clases de fariseos mencione cinco tipos de hipocresía sustituyendo los dos últimos por dos clases de fariseos positivos o que algunas de las acusaciones rabínicas contra los fariseos cuenten con un paralelo casi textual en las acusaciones de Jesús (J. Berajot 14b con Mateo 23, 4). Los mismos esenios de Qumrán son aún más caústicos en sus ataques a los fariseos (PESHER DE NAHUM 2, 710; HODAYOT 4, 11).

En términos generales, los fariseos parecen haber adoptado una postura muy realista, aunque no exenta de espiritualidad, en relación con la guerra. Por sistema se oponían a la misma - aunque eran conscientes de que Dios se impondría sobre Sus adversarios en un enfrentamiento bélico - y procuraban no colisionar con el "statu quo" salvo en la medida en que el mismo se interponía en la práctica de su fe. La legítima defensa era considerada como lícita pero sólo como medida extrema, hasta el punto de que durante el inicio del siglo I d. de $\mathrm{C}$. no optaron en ningun caso por la vía armada para imponer sus puntos de vista. En otras palabras, aceptaban la violencia generada por las instituciones (aunque tendieron a oponerse a la pena de muerte salvo en supuestos muy concretos), reconocían el derecho a la legítima defensa en casos extremos pero repudiaban la violencia revolucionaria. Ciertamente algunos fariseos - como Josefo- se unieron inicialmente al levantamiento contra Roma del año $66 \mathrm{~d}$. de C., pero no es menos cierto que, en su mayoría, no se engañaron, al menos no mucho tiempo, sobre el desenlace final que tendría el conflicto y que, muy posiblemente, sólo deseaban reconducirlo hacia una paz pactada. De hecho, desde muy pronto buscaron la manera de abstenerse de empuñar las armas y de evitar el naufragio de su secta. Así, Rabban Yojanán ben Zakkay no sólo consiguió salir de la Jerusalén sitiada sino que, pronosticando el acceso al trono de Vespasiano, logró que éste le respetara la vida y le permitiera fundar una escuela de estudio y enseñanza de la Escritura en Jamnia. La negativa farisea a embarcarse en aventuras guerreras permitió que el movimiento subsistiera y, posteriormente, se pusiera a la cabeza de la restauración espiritual de Israel. 


\section{LOS SADUCEOS 5}

Al igual que sucede con los fariseos, contamos con noticias relativas a esta secta procedentes de los escritos de Josefo, de los neotestamentarios y de los rabínicos. Sin embargo, los datos de que disponemos resultan mucho más limitados.

Josefo los menciona, por primera vez, junto a los fariseos, en un pasaje al que ya hemos hecho referencia relacionado con Juan Hircano (Ant $13,10,5-6)$. Según el historiador judío, Juan Hircano había sido originalmente simpatizante de los fariseos pero los saduceos consiguieron convertirse en asesores suyos y que se enfrentara con aquellos.

Aparte de este pasaje, Josefo recoge en sus obras cuatro descripciones breves de los saduceos ${ }^{6}$ (Ant 13, 5, 9; 13, 10, 6; 18, 1, 4; Guerra 2, 8, 14). De los detalles suministrados por Flavio Josefo puede deducirse que sólo creían en la Ley de Moisés como Escritura canónica; que rechazaban las tradiciones humanas como vinculantes y, especialmente, las de los fariseos; que no creían en la inmortalidad del alma, ni en la resurrección ni en el infierno; que sostenían la existencia de un libre albedrío y de una responsabilidad del hombre por lo que le aconteciera y que estaban constituidos fundamentalmente por gente de clase alta, lo que eliminaba considerablemente la solidaridad entre ellos.

El Nuevo Testamento confirma el retrato de los saduceos que nos ha llegado a través de Josefo especialmente en lo que se refiere a su negativa a creer en la resurrección y en los ángeles (Hechos 23, 6-8). De hecho, su enfrentamiento teológico con Jesús derivó fundamentalmente de que éste creía en la resurrección (Marcos 12, 18; Lucas 20, 27).

Los saduceos mantenían una fuerte relación con el control del Templo (Hechos 4,$1 ; 5,17$ ) y aunque, muy posiblemente, no todos eran sacerdotes, sí habían sometido a su voluritad el sistema sacerdotal.

Esta vinculación de los saduceos con la vida del Templo así como su pertenencia a la clase alta explica con facilidad su actitud hacia la guerra.

5 Sobre los saduceos, ver: D. GowAN, "The Sadducees» en BBT, pgs. 139-55; J. LIGHSTONE, "Sadducees Versus Pharisees: The Tannaitic Sources» en J. Neusner (ed.), Christianity, Judaism, and Other Greco-Roman Cults: Studies for Morton Smith at 60, Leiden, 1973, vol. 3 , pgs. 206-17; A. Saldarini, Pharisees, Scribes and Sadducees in Palestinian Society: A Sociological Approach, Wilmington, 1988; C. VIDAL MANZANARES, "Saduceos» en Diccionario de las Tres Religiones, Madrid, 1993; Idem, "Saduceos" en Diccionario de Jesús y los Evangelios, Estella, 1994.

6 Una reproducción de los mismos en C. Vidal Manzanares, El Primer Evangelio..., c. 7. 
Parece innegable que colaboraban gustosamente con el poder romano y veían un peligro en cualquier sedición o conflicto bélico. Temían, con muy buen sentido por otro lado, que un incidente de ese tipo acabara extendiéndose a todo el pueblo, cansado por demás del dominio romano, y que finalmente las tropas invasoras acabaran destruyendo el grado de autogobierno de que disfrutaban los judíos, el Templo de Jerusalén y con ello el poder de los saduceos. Es precisamente un pasaje del Nuevo Testamento (Juan 11, 47-50) donde se nos narra el propósito de los saduceos de acabar con Jesús el que recoge a la perfección su psicología política:

«Entonces los principales sacerdotes y los fariseos reunieron el concilio, y dijeron: "¿Qué vamos a hacer? Porque este hombre hace muchas señales. Si le dejamos así, todos creerán en él; Y VENDRAN LOS ROMANOS, Y DESTRUIRAN NUESTRO LUGAR SANTO Y NUESTRA NACION".

Entonces Caifás, uno de ellos, sumo sacerdote aquel año, les dijo: "Vosotros no sabeis nada; ni pensais que nos conviene que un hombre muera por el pueblo, y no que toda la nación perezca"». (Juan 11, 47-50) (Las mayúsculas son nuestras).

Para la casta saducea, cuestiones como la justicia o la equidad parecen haber estado, por regla general, fuera de lugar. Había que salvaguardar el «statu quo» como fuera (aún a costa de derramar sangre inocente) y, en ese sentido, la guerra tenía que ser evitada a cualquier precio. Josefo es tajante al señalar que, en buen número, al estallar la revuelta del $66 \mathrm{~d}$. de $C$. se opusieron a la misma, intentaron calmar al pueblo e incluso acabaron siendo víctimas de los terroristas zelotes. Finos políticos, no se equivocaron en su visión de lo que significaría una revuelta contra Roma. Siguieron existiendo un tiempo como grupo organizado después de la destrucción del Templo de Jerusalén en el año $70 \mathrm{~d}$. de $C$., pero aquel desastre les privó de su base de poder espiritual, social y político. Al fín y a la postre, se vieron desplazados de la vida espiritual por los fariseos y debieron desaparecer como colectivo quizá antes del final del s. I d. de C.

\section{LOS ESENIOS}

Junto a los fariseos y los saduceos existió una secta en la Judea de la época que, aunque desprovista de la importancia de estas dos, presenta rasgos de un notable interés para la historia del periodo. Me estoy refiriendo a los esenios. Donde pudo originarse el nombre es algo sometido todavía a controversia. Para algunos, el mismo no es sino la forma griega 
de «jasya» (piadoso, santo) ${ }^{7}$, mientras que otros lo han relacionado con «'asya" (sanador) ${ }^{8}$, lo que podría encajar con su identificación con los «Zerapeute» (sanadores), una comunidad de vida aislada a la que se refiere Filón (De vida contemplativa, 2 ss) como «adoradores» de Dios.

Las referencias que tenemos sobre los esenios aparecen en una pluralidad de fuentes. Plinio nos da noticia de ellos en su Historia Natural 5, 73 (escrita entre el 73 y el 79 d. de C.), al hacer referencia al Mar Muerto. Filón de Alejandría nos ha dejado dos relatos de los esenios, uno, el más largo, en su obra «Todo hombre bueno es libre», y el otro, más breve, en su apología en favor de los judíos denominada Hypothetica. Josefo se refiere a los esenios en Guerra 2, 119 ss; Ant 18, 18 ss; y Ant 13, 171 ss. Su retrato de los esenios es más detallado que el de Filón y además se centra en testimonios que, al menos en parte, debieron ser de primera mano, ya que en su Vida $10 \mathrm{ss}$, nos habla de que conoció a los esenios cuando era joven. Hipólito se refiere también a los esenios en el noveno libro de su obra Refutación de todas las herejías, escrita en los primeros años del s. $111{ }^{9}$. No hay referencias seguras a los mismos en el Nuevo Testamento y no parece que tuvieran el más mínimo contacto con Jesús.

Sin duda, nuestro conocimiento de al menos un sector de los esenios se ha visto aumentado extraordinariamente como consecuencia de los hallazgos de Qumrán ${ }^{10}$. Empujados al desierto por su oposición a las autoridades del Templo y su desilusión frente a la configuración ideológica del judaísmo tras la victoria de los Macabeos, los sectarios marcharon al desierto, posiblemente, para cumplir la Torah tal y como ellos creían que debía ser obedecida y a la espera de una consumación escatológica del mundo que no se produjo como pensaban. Quizá el grupo se hubiera disuelto de no haber hecho acto de presencia un personaje al que los

\footnotetext{
7 Ver al respecto: J. T. MILIK, Ten Years of Discovery in the Wilderness of Judaea, Londres, 1959 , p. 80; M. BLACK, The Scrolls and Christian Origins, Londres, 1961, pgs. 13 ss; C. VIDAL MANZANARES, Los esenios y los rollos del mar Muerto, Barceiona, 1993 e Idem, Los documentos del mar Muerto, Madrid, 1993.

8 Ver: G. Vermes, “The Etymology of "Essenes"» en Revue de Qumran, 2, 1959-60, pgs. 427 ss.

9 Un análisis extenso de las fuentes mencionadas en C. VIDAL MANZANaRES, Los esenios y los rollos del mar Muerto, Barcelona, 1993 e Idem, El Primer Evangelio, c. 7.

10 La literatura sobre Qumran es extensísima. Al respecto ver: J. A. FITZMYER, The Dead Sea Scrolls: Major Publications and Tools for Study, Missoula, 1977; G. VERMES, The Dead Sea Scrolls, Filadelfia, 1981; P. R. Davies, Qumran, Guildford, 1982; M. DelCor y F. Garcia Martinez, Literatura esenia de Qumran, Madrid, 1982; P. R. CALLAWAY, The History of the Qumran Community, Sheffield, 1988; C. VIDAL MANZANARES, «El origen de la secta del Mar Muerto a la luz de 4QMMT» en Espacio, Tiempo y Forma, Il-3, 1990, pgs. 233-250; Idem, Los esenios y los rollos del mar Muerto, Barcelona, 1993; Idem, Los documentos del mar Muerto, Madrid, 1993; F. GARCÍA y J. TREBOlle, Los hombres de Qumrán, 1994.
} 
documentos de la secta denominan el "Maestro de Justicia». Este imprimió al movimiento una dirección característica que no tendió tanto a mirar hacia atrás — cuando se separaron del sistema de culto de Jerusaléncomo hacia adelante.

El cuartel general de la secta estaba en Jirbet Qumran y allí permanecería durante algo más de dos siglos con un lapso de unos treinta años que el lugar estuvo abandonado (del 31-37 a. de C. al 4 a. de C. aproximadamente). La secta estaba organizada según una jerarquía muy estricta en la que había sacerdotes, levitas, ancianos y los simples monjes. Aunque se reunian en asambleas comunitarias o sesiones de los ha-rabbim (los muchos), lo cierto es que el gobierno efectivo formado por tres sacerdotes y doce laícos. Aparte existían los cargos de mebaqqer (inspector) para controlar diversas áreas de la comunidad y sobre los distintos mebaqquerim, hallamos la figura del paqid (inspector jefe).

Los baños rituales tenían una enorme importancia en la disciplina del grupo ya que aparecían ligados a ideas de pureza ritual. Los restos encontrados en excavaciones evidencian, de hecho, un cuidado escrupuloso en la conservación del agua, algo admirable en el medio desértico donde habitaban.

Las sanciones en el seno de la comunidad eran muy severas e iban desde la reducción de la ración alimenticia a la expulsión en unas condiciones que implicaban casi la muerte segura. La propiedad era comunitaria.

En cuanto a sus creencias, prescindiendo del acento exclusivista propio de la secta, coincidían en buena medida con la teología de los fariseos. También ellos creían en la inmortalidad del alma y en la resurrección, en la existencia de ángeles y de demonios, en el infierno, en una confrontación escatológica final y en la venida del Mesías.

$\mathrm{Su}$ actitud frente a la guerra fue escatológicamente pacifista. En otras palabras, se abstenían de la participación en los conflictos armados pero, a la vez, creían que en el conflicto final entre las fuerzas de la Luz y de las Tinieblas deberían empuñar las armas encuadrados en el primer bando. Uno de sus escritos, la Regla de la Guerra, se ocupa precisamente de describir esta conflagración aunque las referencias aparecen también en otros escritos (1Q Regla de la Guerra 1, 1-8; 15, 1-7; 1Q Hodayot 14, 29-32).

Al producirse el estallido contra Roma del año $66 \mathrm{~d}$. de $\mathrm{C}$. buena parte de los miembros de la secta debieron interpretarlo como la lucha final. Josefo nos informa de que hubo esenios combatiendo en las filas rebeldes y asimismo sabemos que entre los últimos defensores de Masada muy posiblemente también se hallaban sectarios de Qumrán. Su apoyo a la 
revuelta terminó con fatales consecuencias. Con la derrota, sobrevino también el fín del movimiento.

\section{LOS ZELOTES ${ }^{11}$}

La palabra «zelote» o «zelota» es una transcripción del griego «zelotai» que significa, únicamente, "celosos». A su vez, este término helénico no era sino la traducción de la autodenominación que se daban los componentes de este colectivo: "qannaim» (celosos, en hebreo) o «qananayya» (celosos, en arameo). El nombre implicaba un celo religioso destinado a preservar el honor del Dios de Israel contra cualquier persona, cosa o situación que, a juicio de los zelotes, lo menoscabara. A diferencia de los grupos mencionados hasta ahora, los zelotes consideraban la cuestión de la guerra como algo esencial a su actividad espiritual. No en vano tenían como modelo a Finees, un nieto de Aarón, que no había dudado, llevado por el celo de Dios, en traspasar de una lanzada a un israelita y a una pagana mientras se encontraban fornicando (Números 25, 7-13). Con este predecente, no debería extrañarnos que los zelotes mataran a los judíos que se casaban con no-judías sin ningun tipo de proceso previo (Sanhedrín 9, 6).

La primera referencia histórica a los zelotes como partido articulado se nos da en relación con los secuaces de Menahem que, en el año $66 \mathrm{~d}$. de C., intentaron hacerse con el control de la guerra contra Roma (Guerra II, 441). El término es utilizado también por Josefo para hacer referencia a los rebeldes jerosilimitanos del invierno del 66-67 d. de C. (Guerra II, 651) y a los seguidores de Juan de Giscala que se hicieron con el área del Templo en el $67 \mathrm{~d}$. de $\mathrm{C}$. De la misma manera, las fuentes rabínicas situan las actividades de los zelotes en el periodo de la guerra contra Roma (Abot de Rabbi Nathan 6,8).

Estas noticias son evidentemente correctas porque desde la revuelta de Judas el Galileo (que debió de tener lugar durante la infancia de Jesús), los judíos intentaron tratar las tensiones con Roma pacíficamente y, como

11 Sobre los zelotes, ver: O. CullmanN, Jésus et les révolutionaires de son temps, Neuchâtel, 1971; G. BAUMBACH, «Zeloten und Sikarier», en TLZ, 90, 1965, cols. 727-740; Idem, «Die Zeloten» en BiLit, 41, 1968, pgs. 2-25; S. APPLEBAUM, «The Zealots: the case for revolution» en JRS, 66 , 1971, pgs. 155-170; J. HitTE, Massada, Jerusalén, 1973; H. GuevarA, Ambiente político del pueblo judío en tiempos de Jesús, Madrid, 1985; Y. YADIN, Masada: la fortaleza de Herodes y el último bastión de los Zelotes, 1986 ( $3^{\mathrm{a}}$ ed.); M. HENGEL. The Zealots, Edimburgo, 1989; C. VIDAL MANZANARES, «Guerra» y «Zelotes» en Diccionario de Jesús y los Evangelios, Estella, 1994. 
mucho, recurriendo a lo que ahora denominaríamos «acciones no-violentas». Fue sólo la política de los últimos procuradores romanos y el estallido del conflicto final contra Roma lo que provocó el nacimiento de los zelotes. Los mismos, por lo tanto, no existieron en vida de Jesús (salvo el caso de Judas el Galileo al que nos referiremos enseguida) y esa circustancia por si sola - pero a la que se pueden unir muchas más - imposibilita la adscripción del mismo a ese movimiento como han hecho en forma carente de base y más ligada a la especulación que al análisis desapasionado de las fuentes autores como S. G. F. Brandon ${ }^{12}$.

Con todo, la historia de este colectivo ha venido opacada por una referencia contenida en Josefo (Guerra II, 118; Ant XVII, 9, 23) en el sentido de que Judas el galileo, que se sublevó contra los romanos hacia el $6 \mathrm{~d}$. de C., fue el fundador de la "cuarta filosofía», la de los zelotes. Dado que Josefo acostumbra a denominar a los zelotes, «bandidos» y términos similares, algunos autores han intentado trazar una línea ininterrumpida de existencia de los zelotes desde el año $6 \mathrm{~d}$. de C., hasta la toma de Masada. Como ya pudimos ver en la primera parte de esta obra, tal posibilidad no se puede mantener. La muerte de Judas el galileo significó el final de su movimiento (Hechos 5,37 ) y no tenemos noticias de nada similar - con la excepción de la sublevación del "engañador egipcio» que, no obstante, no parece haber sido un zelote- hasta el año $66 \mathrm{~d}$. de C. en el que, correctamente, Josefo habla de ellos. Intentar interpretar como zelotes a todos los bandoleros - calificados como tal por Josefo-que aparecieron durante los sesenta años intermedios no resulta de recibo como ha demostrado recientemente un autor hispano ${ }^{13}$. Hoy por hoy, por lo tanto, no creemos que se pueda afirmar que existieran zelotes en el periodo que va de la muerte de Judas el galileo a los primeros años posteriores a la muerte de Jesús y, muy probablemente, al $66 \mathrm{~d}$. de $\mathrm{C}$.

La teología específica de los zelotes no parece haber sido muy distinta de la de los fariseos pero, a diferencia de éstos, ellos se manifestaron partidarios de iniciar una acción armada contra Roma que, pensaban, sería respaldada por Dios. Precisamente por ello, eran contrarios al pago del tributo al emperador y a los matrimonios mixtos. A su uso de la fuerza

12 S. G. F. Brandon, Jesus and the Zealots, Manchester, 1967. Refutaciones de sus aventuradas especulaciones en M. HENGEL, The Zealots...; Idem, Was Jesus a Revolutionist, Filadelfia, 1971; O. Cullmann, Jesus and the Revolutionaries, Nueva York, 1970; H. Guevara, O.c; y C. VIDAL MANZANARES, El judeo-cristianismo palestino....

13 Ver: H. Guevara, O.c. Hemos contribuido a la misma tesis en C. Vidal Manzanares, EI Primer Evangelio... e Idem, El judeo-cristianismo palestino en el s. I: de Pentecostés a Jamnia, Madrid, 1994. 
armada ligaron aspectos típicos de la violencia revolucionaria como quemar los registros de propiedad, asesinar a miembros de la clase alta, etc. Su postura llevó finalmente a la nación a la ruina ( $y$, en este sentido, es comprensible la postura contraria que adopta Josefo), pero tanto la incapacidad de la clase gobernante judía como la rapacidad romana habian abierto las puertas a que sus posturas radicales (hoy quizá diríamos «fundamentalistas») fueran aceptadas por buena parte de la población ${ }^{14}$.

\section{LOS JUDEO-CRISTIANOS ${ }^{15}$}

Al lado de los grupos anteriores debemos incluir a otro que tendría una enorme importancia en la configuración del cristianismo como fe universal. Me refiero al colectivo fundado por Jesús y continuado tras la muerte de éste c. $30 \mathrm{~d}$. de C. Resulta indiscutible que para Jesús ${ }^{16}$, la época en que vivió fue considerada como central para la historia universal. Iniciada con la recepción del Espíritu Santo por Jesús en el momento de su bautismo ( $Q 3,16$ b), en ella había venido el Reino ( $Q$ 17, 20-21). En la misma se enfrentaban de manera decisiva las fuerzas del bien y del mal pero su origen era puramente espiritual. Quizá alguien esperaría ver a Jesús culpando de los males de su tiempo al imperialismo romano o a la pésima distrìbución de la riqueza en la Palestina de su época, pero apenas puede encontrarse una imagen más lejana de la realidad reflejada en las fuentes históricas que ésa. Para Jesús, el adversario número uno era el Diablo y sus huestes demoníacas. La misma política es descrita tanto en $Q$ como en los cuatro Evangelios canónicos como un sistema controlado por Satanás que ostenta el dominio de todos los reinos de la tierra $(Q 4,5-7)$. La segunda gran fuerza del mal que Jesús percibe como enemiga suya es la auto-justificación religiosa $(Q 11,46 ; 11,43 ; 11,52 ; 11,37-39 a ; 11,42$;

\footnotetext{
14 Se ha relacionado el término «sicario" (el que lleva la «sica» o puñal) con los zelotes. La palabra, en su sentido estricto, parece haberse referido propiamente a asesinos y extorsionadores no impulsados necesariamente por razones políticas. Seguramente eso explica que Josefo la refiera a los zelotes, sin duda, por su contenido denigratorio. De nuevo, la palabra no puede ser identificada, sin más, con los zelotes.

15 La obra más completa sobre el tema sigue siendo nuestro El judeo-cristianismo palestino en el s. l, Madrid, 1994. Un acercamiento muy similar en lo que al desarrollo histórico se refiere aunque más breve y dejando de lado las cuestiones sociales, institucionales e ideológicas en $R$. PfiTz, Nazarene Jewish-Christianity, Jerusalem-Leiden, 1988.

16 Las referencias a la enseñanza de Jesús aparecen aquí circunscritas al Documento o Fuente $Q$, ya que éste es la única colección de enseñanzas suyas redactada sin dudas en territorio palestino (es mucho más controvertido el caso de Mateo o de Juan). El texto completo de $Q$ puede hallarse en C. VIDAL Manzanares, El Primer Evangelio: el Documento Q, Madrid, 1994.
} 
11, 43-54). Dado que Jesús insistía en un análisis puramente espiritual de la realidad centrado en la existencia de una lucha feroz entre el mal (Satanás y sus acólitos) y el bien (Jesús y el Reino iniciado por él), lo esencial no podía ser el cambio de estructuras sociales, la alteración del orden político existente o la toma de las armas para implantar una teocracia. Más bien aquello a lo que llamaba a todos era a tomar partido en semejante confrontación por él ya que no hacerlo sólo puede terminar en desastre (Q 15, 1-7 y $Q$ 15, 8-10). Los que se excusaran, los indiferentes, los apáticos se quedarían fuera y padecerían el suplicio del infierno $(Q$ $10,15 ; 12,5)$.

Ahora bien, ¿qué significaba seguir a Jesús en términos prácticos? No parece que Jesús fuera un legalista al uso. Ciertamente, insistió en la importancia de orar y enseñó a sus discípulos como hacerlo $(Q 11,1-4 ; 11$, $5-13)$, incluso dio por bueno y vigente el contenido de la Torah $(Q 16,17)$. Pero, al lado de esto, se manifestó intolerablemente flexible en lo que al sábado se refería $(Q 14,1-6)$ y subordinó el mandato del diezmo a las cosas más importantes de la Ley de Dios, Su justicia y Su amor (Q 11, 42). Lo primero que debía caracterizar a los seguidores de Jesús era una vivencia del Espíritu Santo que tendría como resultado manifestaciones de poder, pero de un poder espiritual, pneumático. Lo segundo, una fe absoluta en el cuidado divino (Q 12, 22 ss; 12, 31). Lo tercero, una vida de amor, entendiendo éste de una manera muy específica. Para empezar, el amor debería hacerse extensivo a los enemigos $(Q 6,27)$.

No cabe duda de que esta enseñanza es propia de Jesús y no se da en ninguna otra enseñanza moral. Como mucho, tanto judíos como no-judíos habían llegado a la fórmula, por otro lado admirable, de "no hagas a otro lo que no deseas que te hagan a ti». Pero la enseñanza de Jesús iba mucho más allá. Implicaba amar al enemigo, hacer el bien a los que nos aborrecen, bendecir a los que nos maldicen, orar por los que nos denigran y renunciar a todo tipo de violencia incluida la defensiva (Mateo 5, 44-48). Se podrá argumentar, y así lo hizo en su día J. Klausner ${ }^{17}$, que «esta ética individualista y extremista... ni la sociedad, ni el Estado, ni la nación estaban en condiciones de soportar(la)». Tal objeción es válida y, a juzgar por las fuentes, se corresponde con una interpretación rigurosamente exacta de la enseñanza de Jesús. Ciertamente, no hay Estado que pueda mantenerse en el principio de ofrecer la otra mejilla, orar por los enemigos o bendecir al que lo denigra. Pero, con todo, ése es un tema

17 J. KLAUSNER, Jesús de Nazaret, Buenos Aires, 1971, p. 405. 
que no debió preocupar a Jesús, quien consideraba que todos los gobiernos estaban bajo el control del Diablo (Q 4, 5 ss).

El que aceptara a Jesús tendría que llevar una vida con arreglo al carácter del Dios predicado por Jesús, un Dios que era bueno y misericordioso incluso con los malos ( $Q 6,35$ y $Q 6,36)$, y cuya mayor prueba de amor era que Jesús había venido en busca de los que se habían extraviado ( $Q 15,4-7$ y $Q 15,8-10)$. El uso de la violencia quedaba, por lo tanto, descartado.

En el tiempo intermedio, de duración indeterminada, entre el inicio de su predicación y su regreso como Hijo del hombre para juzgar al mundo ${ }^{18}(Q$ 13, 18-21) los seguidores de Jesús deberían abstenerse igualmente del recurso a la violencia. La carta de Santiago, un escrito judeo-cristiano de los años 40-50, atribuido a uno de los hermanos de Jesús constituye un exponente ideal de lo que aquí mencionamos. El rechazo a la violencia -aún más, la norma positiva de amar al prójimo como a uno mismo- es colocado a la altura de los mandatos contenidos en la Torah (Santiago 2, 8-11). Sólo de la paz puede esperarse el brote de la justicia $(2,18)$ y la injusticia en ningun caso puede ser respondida con violencia sino con mansedumbre y confianza en que la Segunda Venida de Jesús, esta vez como Juez de vivos y muertos, implicará un cambio de todo $(5,4-8)$. Mientras tanto el consuelo - y la alegria- brota de la estrecha relación con Dios y de la vivencia carismática en el seno de la comunidad (5, 10-20).

Por lo que sabemos, los judeo-cristianos se mantuvieron fieles a esta visión de repulsa frente a la guerra durante la contienda iniciada en el año $66 \mathrm{~d}$. de $\mathrm{C}$. Lejos de enzarzarse en el conflicto, los pertenecientes a la comunidad jerosilimitana marcharon a Pella desde donde esperaron el final del conflicto ${ }^{19}$.

\section{CONCLUSIÓN}

El judaísmo del Segundo Templo constituia ideológicamente una realidad muy diversa. Esta quedó reflejada asimismo de manera obvia en la actitud hacia la guerra que tuvieron las sectas que lo integraban. Aquellas que se sumaron al esfuerzo bélico, bien provocándolo (los zelotes) bien

18 En este mismo sentido, ver: F. F. Bruce, New Testament History, Nueva York, 1980, p. 177.

19 He estudiado este tema con anterioridad en C. VIDAL MANZANARES, El judeo-cristianismo palestino en el s. I: de Pentecostés a Jamnia, Madrid, 1994 e Idem, «Pella revisitada» en Espacio, Tiempo y Forma, Serie II, 6, 1993, pp. 467-476. 
incorporándose una vez que llegaron a la conclusión de que el mismo tenía categorías escatológicas (esenios de Qumrán) serían arrastradas por el desastre y desaparecerían en el mismo. De hecho, no volvemos a saber de las mismas en la historia del Israel ulterior ${ }^{20}$. De las que se negaron a dejarse llevar por la oleada de patriotismo en el año $66 \mathrm{~d}$. de C. bien por temor a perder su posición privilegiada (saduceos), bien por pesimismo frente a una posible victoria (fariseos), bien por pacifismo radical (judeo-cristianos), podemos decir que sobrevivieron en su totalidad a la calamidad del 70 d. de $\mathrm{C}$. Pese a todo, su evolución resultó diferente. Los saduceos habían logrado no verse aniquilados por la derrota pero carentes de la base de su poder - el control sobre el ritual del Templo - una vez que resultó obvio que la reconstrucción del santuario jerosilimitano no sería inmediata se fueron viendo relegados de la vida espiritual judía por los fariseos hasta desaparecer por completo. No tuvieron tan mala fortuna los judeo-cristianos. Pese a ser expulsados, c. 80-90 d. de C., del seno de Israel en virtud de la "birkat ha-minim", seguirían existiendo como colectivo bien diferenciado al menos hasta el s. IV d. de $\mathrm{C}$. En cuanto a su vertiente gentil, el cristianismo no sólo no iba a desaparecer sino que pronto iba a desplazar a los misioneros fariseos en la captación de las voluntades de los antaño paganos. De los fariseos sabemos que, tras solucionar sus propias querellas internas en favor fundamentalmente del sector hil.lelita, se convirtieron en la guía de Israel, cuya enseñanza quedaría plasmada en la Mishnah y los Talmudim.

Pocos aspectos, si es que alguno, resultaron, por lo tanto, más decisivos que su visión de la guerra para explicar la supervivencia y la desaparición de los sectores que integraban espiritualmente el pueblo de Israel al final del período del Segundo Templo. Los que supieron discernir que aquel conflicto no venía inspirado por el deseo de Dios de liberar a Israel del dominio gentil sobrevivieron, los que tomaron sus propios deseos como un impulso divino de liberación quedaron borrados de la faz del pueblo.

20 A menos que consideremos a los karaítas medievales como una prolongación de los esenios. Al respecto, véase: C. VIDAL MANzanares, Los esenios y los rollos del mar Muerto, Barcelona, 1993, último capítulo. 